\title{
HOMOGENEOUS MINIMAL SURFACES IN EUCLIDEAN SPHERES WITH FLAT NORMAL CONNECTIONS
}

\author{
KICHOON YANG
}

\begin{abstract}
We classify, up to congruence, homogeneous minimal surfaces in Euclidean spheres with flat normal connections. The parameter varieties in the space of contact invariants of E. Cartan are computed for all codimensions.
\end{abstract}

0. Introduction. In studying minimal submanifolds of Riemannian manifolds one often encounters Euler-Lagrange type differential equations. In the case of minimal surfaces in the Euclidean $n$-sphere $S^{n}$ (or minimal submanifolds in homogeneous Riemannian manifolds in general) the partial differential equations arise geometrically as structure equations which give relations among contact invariants of $\mathrm{E}$. Cartan associated with the bundle of Frenet frames. The equations thus obtained are quite difficult to solve completely and one looks for partial solutions by imposing suitable restrictions upon the class of minimal submanifolds to be investigated.

We now briefly describe our result. Take a minimal surface $M$ in $S^{2+p}$ and assume that the induced connection on the normal bundle is flat. This allows us to simultaneously diagonalize all $p$ second fundamental forms and quickly leads to the construction of Frenet frames on $M$. Further, assuming that $M$ is homogeneous, the resulting structure equations are solved completely. This gives, up to congruence, the classification of homogeneous minimal surfaces in $S^{2+p}$ for all $p$ with flat normal connections.

All maps and objects are assumed to be smooth and the following index convention will be used throughout:

$$
\begin{aligned}
& 1 \leqslant \alpha, \beta, \lambda, \ldots \leqslant 2+p \\
& 1 \leqslant i, j, k, \ldots \leqslant 2, \\
& 3 \leqslant a, b, c, \ldots \leqslant 2+p .
\end{aligned}
$$

1. Higher order moving frames. Let $f: M^{2} \hookrightarrow S^{2+p}$ be a surface in the Euclidean sphere of dimension $2+p$.

$S^{2+p}$ with the standard metric is realized as a Riemannian homogeneous space $S O(3+p) / S O(2+p)$. We write $\left(e_{0}, e_{i}, e_{a}\right)$ to denote elements of $S O(3+p)$. They are orthonormal frames on $S^{2+p}$. The projection $\pi: S O(3+p) \rightarrow S^{2+p}$ is given by $\pi\left(e_{0}, e_{i}, e_{a}\right)=e_{0}$.

Received by the editors March 23, 1983.

1980 Mathematics Subject Classification. Primary 53A10. 
Let $f^{-1} S O(3+p)$ denote the pull-back bundle over $M$. The bundle of first order moving frames of $f$, contained in $f^{-1} S O(3+p)$ and denoted by $L_{1}$, is defined by the condition that the vectors $\left(e_{a}\right)$ are orthogonal to the surface. It is easily seen that $L_{1}$ is a principal bundle over $M$ with the structure group $S(O(2) \times O(p))$. $L_{1}$ splits into the orthogonal sum $O(M) \oplus O^{\perp}(M)$, where $O(M)$ is the bundle of orthonormal frames over $M$ and $O^{\perp}(M)$ is the normal bundle over $M$.

Let $\left(\theta^{\alpha}, \omega_{\beta}^{\alpha}\right)$ denote the Maurer-Cartan form of $S O(3+p)$. It is $\mathcal{O}(3+p)$-valued and its components are the left-invariant 1 -forms on $S O(3+p)$ satisfying

$$
d e_{0}=\theta^{\alpha} \otimes e_{\alpha}, \quad d e_{\beta}=\omega_{\beta}^{\alpha} \otimes e_{\alpha} \oplus \theta_{\beta} \otimes e_{0},
$$

where $\omega_{\beta}^{\alpha}+\omega_{\alpha}^{\beta}=0, \theta^{\alpha}+\theta_{\alpha}=0$. Exterior differentiation of (1.1) gives

$$
d \theta^{\alpha}=-\omega_{\beta}^{\alpha} \wedge \theta^{\beta}, \quad d \omega_{\beta}^{\alpha}=-\omega_{\gamma}^{\alpha} \wedge \omega_{\beta}^{\gamma}+\theta^{\alpha} \wedge \theta^{\beta} .
$$

Observe that $\left(\theta^{a}\right) \equiv 0$ on $L_{1}$. Thus $d f=d e_{0}=\theta^{1} \otimes e_{1} \oplus \theta^{2} \otimes e_{2}$. Differentiating both sides of the equations $\left(\theta^{a}\right) \equiv 0$, and using (1.2) we obtain

$$
\omega_{j}^{a} \wedge \theta^{j}=0 \text {. }
$$

By Cartan's lemma we may set, for some functions $A_{j k}^{a}=A_{k j}^{a}$ on $L_{1}$,

$$
\omega_{j}^{a}=A_{j k}^{a} \theta^{k} \text {. }
$$

Fix a first order moving frame field $u: M \rightarrow L_{1}$. Write $u^{*} \omega_{j}^{a}=X_{j k}^{a} u^{*} \theta^{k}$, for some functions $\left(X_{j k}^{a}\right)$ on $M$. Suppose $\bar{u}: M \rightarrow L_{1}$ is given by $\bar{u}=u \cdot g$ for a smooth map $g$ : $M \rightarrow S(O(2) \times O(p))$. Let $\left(\bar{X}_{j k}^{a}\right)$ be functions on $M$ so that $\bar{u}^{*} \omega_{j}^{a}=\bar{X}_{j k}^{a} \bar{u}^{*} \theta^{k}$.

Some matrix multiplications show that the action of $S(O(2) \times O(p))$ on $\left(X_{j k}^{a}\right)$ is given by

$$
\bar{X}_{j k}^{a}=\left(b^{-1}\right)_{b}^{a} X_{m n}^{b} a_{j}^{m} a_{k}^{n},
$$

where $g=(a, b) \in S(O(2) \times O(p))$.

We now assume that $p=1$ and derive the structure equations for minimal surfaces in $S^{3}$. Using the action (1.5) we diagonalize the second fundamental tensor and define the bundle of second order moving frames over $M$, denoted by $L_{2}$, by the following condition:

$$
\omega_{1}^{3}=k \theta^{1}, \quad \omega_{2}^{3}=\bar{k} \theta^{2} .
$$

Minimality of $M$ requires that $\bar{k}=-k$.

We note that if $k \equiv 0$, then $M$ is an open submanifold of a great two-sphere. From now on we exclude this case from our discussion.

Assuming that $k>0$ and choosing an orientation class we identify $L_{2}$ with $M$. So $\boldsymbol{\theta}^{1}$ and $\boldsymbol{\theta}^{2}$ form a coframe field on $\boldsymbol{M}$.

Set $d k=k_{1} \theta^{1}+k_{2} \theta^{2}$. Then exterior differentiation of (1.6) together with (1.2) imply that

$$
\omega_{2}^{1}=(1 / 2 k)\left(k_{1} \theta^{2}-k_{2} \theta^{1}\right) ;
$$

$\omega_{2}^{1}$ is the Levi-Civita connection form.

We also have

$$
d \omega_{2}^{1}=\left(1-k^{2}\right) \theta^{1} \wedge \theta^{2}
$$


$1-k^{2}$ is the Gaussian curvature. Hence the Gaussian curvature of a minimal surface in $S^{3}$ is bounded by 1 . (This is true in any codimension.)

Differentiating the right-hand side of (1.7) and using (1.8), we obtain

$$
4 k^{2}\left(k^{2}-1\right)=3\left(k_{1}^{2}+k_{2}^{2}\right)-2 k\left(k_{11}+k_{22}\right)
$$

where $d k_{i}=k_{i j} \theta^{j}$.

We now go back to the general case, that is, $p$ is arbitrary.There are $p$ second fundamental tensors, and looking at the action (1.5) finding a suitable normal form seems difficult. (See [3].)

We assume that the second fundamental tensors are simultaneously diagonalizable. As we shall see shortly, this amounts to assuming that the normal connection is flat:

$$
\omega_{1}^{a}=k^{a} \theta^{1}, \quad \omega_{2}^{a}=-k^{a} \theta^{2} .
$$

It follows that

$$
d \omega_{b}^{a}=-\omega_{c}^{a} \wedge \omega_{b}^{c}
$$

Thus the normal connection (i.e., the induced connection on $\left.O^{\perp}(M)\right)$ is flat. $\left(\left(\omega_{b}^{a}\right)\right.$ is the connection matrix of the normal connection.)

Differentiating the Levi-Civita connection form we obtain

$$
d \omega_{2}^{1}=\left(1-\sum_{a}\left(k^{a}\right)^{2}\right) \theta^{1} \wedge \theta^{2}
$$

Once again, if $\left(k^{a}\right) \equiv 0$, then $M$ is an open submanifold of a two-dimensional equator. From now on we exclude this possibility.

2. The main result. The discussion from the last section implies that the totality of minimal surfaces in $S^{2+p}$ with the flat normal connections arise as integral surfaces of the following Pfaffian system on $S O(3+p)$ with the specified independent variables $\theta^{1}$ and $\theta^{2}$ :

$$
\sum:\left\{\theta^{a}=0, \omega_{1}^{a}=k^{a} \theta^{1}, \omega_{2}^{a}=-k^{a} \theta^{2}, a=3,4, \ldots, 2+p\right\} .
$$

We close the system and write the quadratic equations modulo the system

$$
\begin{aligned}
& 2 k^{a} \theta^{2} \wedge \omega_{2}^{1}-k^{b} \theta^{1} \wedge \omega_{b}^{a}-\theta^{1} \wedge d k^{a} \equiv 0, \\
& 2 k^{a} \theta^{1} \wedge \omega_{2}^{1}+k_{b} \theta^{2} \wedge \omega_{b}^{a}+\theta^{2} \wedge d k^{a} \equiv 0 .
\end{aligned}
$$

The system is in normal form and a quick counting shows that it is overdetermined.

We prolong the system by setting

$$
\omega_{2}^{1}=t_{1} \theta^{1}+t_{2} \theta^{2}, \quad \omega_{b}^{a}=n_{b 1}^{a} \theta^{1}+n_{b 2}^{a} \theta^{2}, \quad d k^{a}=k_{1}^{a} \theta^{1}+k_{2}^{a} \theta^{2} .
$$

We now make the assumption that $M$ is homogeneous. This allows us to set $t_{j}, n_{b j}^{a}, k^{a}$ equal to some constants, since homogeneous surfaces do not admit nonconstant contact invariants [4, p. 42]. Thus $\left(d k^{a}\right) \equiv 0$.

Substituting the remaining equations in (2.3) into (2.2) we have

$$
\begin{aligned}
& 2 k^{a} t_{1}+n_{b 2}^{a} k^{b}=0, \\
& 2 k^{a} t_{2}-n_{b 1}^{a} k^{b}=0 .
\end{aligned}
$$


Multiply each equation in (2.4a) by $k^{a}$ and sum over the index $a$ :

$$
2 t_{1} \sum_{a}\left(k^{a}\right)^{2}+\sum_{a, b} n_{b 2}^{a} k^{b} k^{a}=0 .
$$

Similar operations on $(2.4 \mathrm{~b})$ give

$$
2 t_{2} \sum_{a}\left(k^{a}\right)^{2}-\sum_{a, b} n_{b 1}^{a} k^{b} k^{a}=0 .
$$

Noting that $\left(n_{b j}^{a}\right)$ are skew-symmetric in $a, b,(2.5 \mathrm{a})$ and (2.5b) imply that $t_{1}=t_{2}=0$. This gives

$$
\omega_{2}^{1}=0 .
$$

Differentiating both sides of (2.6) we see that $1-\Sigma_{a}\left(k^{a}\right)^{2}=0$. That is, $M$ is flat. (2.4a) and (2.4b) become

$$
n_{b 1}^{a} k^{b}=0, \quad n_{b 2}^{a} k^{b}=0 .
$$

Fixing the vector $\left(k^{b}\right) \in S^{p-1}$, let $\left(l_{b j}^{a}\right)$ denote any solution to (2.7):

$$
\omega_{b}^{a}=l_{b j}^{a} \theta^{j} \text {. }
$$

Differentiating both sides of the above equations and using the homogeneity assumption again we obtain

$$
l_{c 1}^{a} l_{b 2}^{c}=l_{c 2}^{a} l_{b 1}^{c} \text {. }
$$

The prolonged system is on $S O(3+p) \times \mathbf{R}^{p^{2}}$ and given by the following equations:

$$
\begin{aligned}
& \sum_{a}\left(k^{a}\right)^{2}=1, \quad n_{b 1}^{a} k^{b}=0, \\
& n_{b 2}^{a} k^{b}=0, \quad n_{c 1}^{a} n_{b 2}^{a}=n_{c 2}^{a} n_{b 1}^{a}
\end{aligned}
$$

and

$$
\begin{aligned}
& \theta^{a}=0, \quad \omega_{1}^{a}=k^{a} \theta^{1}, \\
& \omega_{2}^{a}=-k^{a} \theta^{2}, \quad \omega_{b}^{a}=n_{b 1}^{a} \theta^{1}+n_{b 2}^{a} \theta^{2} .
\end{aligned}
$$

The above system is completely integrable.

Let $V$ denote the algebraic variety in $\mathbf{R}^{p^{2}}$ defined by the system of polynomial equations in (2.10). We have

THEOREM. The totality of nonequatorial homogeneous minimal surfaces in $S^{2+p}$ with the flat normal connections is parametrized by the algebraic variety $V \subset \mathbf{R}^{p^{2}}$.

\section{REFERENCES}

1. E. Cartan, Théorie des groupe finis et continues et la géométrie différentielle traitées par la méthode du repère mobile, Gauthier-Villars, Paris, 1937.

2. Les systèmes différentiels extérieurs et leurs applications géométriques, Hermann, Paris, 1945.

3. Wu-Yi Hsiang, Remarks on closed minimal submanifolds in the standard Riemannian m-sphere, J. Differential Geom. 1 (1967), 257-267.

4. G. Jensen, Higher order contact of submanifolds of homogeneous spaces, Lecture Notes in Math., vol. 610, Springer-Verlag, Berlin, 1977.

5. K. Yang, Prolongations of exterior differential systems, preprint 1982.

Department of Mathematics, Arkansas State University, State University, Arkansas 72467 